\title{
PHYSIOLOGICAL AND BIOCHEMICAL STUDIES ON THE LONGEVITY OF ASPERGILLUS ORYZAE CONIDIA STORED UNDER VARIOUS ENVIRONMENTAL CONDITIONS ${ }^{1}$
}

\author{
MITSUKO TOKORO* and TOMOMICHI YANAGITA \\ Institute of Applied Microbiology, University of Tokyo, Tokyo \\ and \\ *Department of Biology, Ochanomizu University, Tokyo \\ Received May 25, 1966
}

The longevity of fungal spores is known to be affected greatly by chemical and physical factors $(1,2)$. Among various physical factors, temperature and humidity have been widely investigated using various species of fungal spores. Other environmental factors, such as gaseous and liquid environments, also affect the spore longevity. In spite of these extensive physiological observations on the longevity of various fungal spores, biochemical studies on the mechanism of deterioration of spores seems to have been largely neglected.

In the present report, the effects of various environmental factors and the physiological state on the longevity of Aspergillus oryzae conidia are described. High temperature, high humidity and anaerobiosis were found to shorten the longevity of the powdery conidia. When washed conidia were incubated in water, they deteriorated as slowly as powdery conidia stored in moist air. Biochemical investigation led us to the conclusion that the course of deterioration of conidia samples stored in moist air and in water may be different in the initial step.

\section{MATERIALS AND METHODS}

Organism. Conidia of Aspergillus oryzae obtained from KojiyaSanzaemon Co., Kyoto, were used throughout. This material is the seedconidia ("tanekoji") used for the industrial preparation of "koji". A mass of the freshly prepared dry conidia was packed in a polyethylene bag, placed in a can containing silica gel for desiccation, and conidia were stored at $4^{\circ}$. Under these storage conditions, the conidia remained highly stable for years without deterioration in the activity of germination.

Test for germination. The germination rate was determined as a function of the length of storage of conidia. The germination medium

1 Present investigation was supported in part by grant from the Waksman Foundation of Japan. 
employed was peptone- $\mathrm{NH}_{4} \mathrm{NO}_{3}$-glucose medium (3) containing $1.5 \%$ agar. The germinative capacity of the conidia was tested by the 'time course method' involving spreading of the test conidia on agar medium and measuring germination rate at time intervals or by the 'end point method' involving determination of germination rate at the 5.5th $\mathrm{hr}$ of cultivation. The untreated conidia stored dry at $4^{\circ}$ were employed as the control in the determination of germination rate. In the control run, $95 \%$ of the conidia usually germinated after $5.5 \mathrm{hr}$ and $50 \%$ after $3.8 \mathrm{hr}$ of cultivation. The germination rate of each conidia sample was expressed as per cent of control.

Storage conditions. (1) Storage under gas of different humidities. The unwashed powdery conidia (about $100 \mathrm{mg}$ dry weight) were stored under various gaseous conditions keeping the layer of the conidia mass as thin as possible. Vessels employed were mainly Thunberg tubes containing test conidia in side arms. The relative humidity in the vessel was kept at the desired value by $3 \mathrm{ml}$ of humidity-regulating sulfuric acid solution (4) in the main chamber. The gas phase was exchanged with a gas mixture of known composition after repeated evacuations and refillings with the gas.

(2) Storage in water. When the conidia were stored in water (containing $50 \mu \mathrm{g} / \mathrm{ml}$ streptomycin, $\mathrm{pH} 6.0$ ), conidia were washed thoroughly with cold water and suspended at a cell density of $0.5 \mathrm{mg}$ dry weight per $\mathrm{ml}$. The suspension was incubated on a shaker.

In the present experiments, incubation temperature was $30^{\circ}$ unless otherwise stated.

Measurement of respiratory activity. Employing Clark's oxygen electrode (Yellow Spring Instrument Co., Antioch, Ohio) the consumption of oxygen dissolved in water was measured. A vessel designed by Dr. T. Nakamura (Department of Biochemistry and Biophysics, University of Tokyo) contained $3.5 \mathrm{ml}$ of conidia suspension. The measurement was performed under constant stirring with a magnetic stirrer at $30^{\circ}$ with a Hitachi Recorder QPD 33 (input $1 \mathrm{mv}$ ).

Washed conidia were suspended in water at a density of $1 \mathrm{mg}$ dry conidia per $\mathrm{ml}$ and after the measurement of endogenous respiration for $20 \mathrm{~min}$ in this system, the glucose respiration was measured by adding glucose at a concentration of $0.28 \%$. No difference in the activity was noticed either in $0.1 \mathrm{~N}$ phosphate buffer $(\mathrm{pH} 6.0)$ or in water.

The calculation of $\mathrm{Q}_{2}(\mu \mathrm{l} \mathrm{O} / \mathrm{hr} / \mathrm{mg}$ dry conidia) was based on an assumption that the concentration of oxygen saturated with air at $30^{\circ}$ under 1 atmospheric pressure is $240 \mu$ moles per liter of distilled water and this value is not affected by the addition of small amounts of cells, salts and sugar.

Measurement of mannitol content in conidia. Washed conidia of known amount (10-30 $\mathrm{mg}$ dry weight) was extracted 3 times with $1 \mathrm{ml}$ of water at $100^{\circ}$ for $15 \mathrm{~min}$ and the combined extract was concentrated in 
vacuo to $1 \mathrm{ml}$. The concentrate was assayed for mannitol content employing essentially the mannitol dehydrogenase method of HoRIKosHI et al. (5).

To prepare cell-free enzyme solution for the mannitol assay, $1 \mathrm{~g}$ of conidia was sonicated with a $10 \mathrm{kc}$ sonicator (Toyoriko Co., Tokyo) in the presence of glass beads $(0.3 \mathrm{~mm}$ average diameter), and the supernatant of the disintegrate was salted out with $70 \%$ saturation of ammonium sulfate. The precipitates were dissolved in $10 \mathrm{ml}$ of $0.1 \mathrm{M}$ acetate buffer $(\mathrm{pH} 6.0)$ and dialysed against the same buffer. This enzyme preparation showed activities of NAD-linked mannitol dehydrogenase, NAD-linked trehalase and NADlinked erythritol dehydrogenase (activity ratio being $1.00: 0.33: 0.27$ ).

For the measurement of mannitol content in the conidia extract prepared as described above, a reaction mixture composed of $0.1 \mathrm{M} \mathrm{NAD} 0.05 \mathrm{ml}$, conidia extract $0.05 \mathrm{ml}$, enzyme solution $0.1 \mathrm{ml}$ ( 170 units), and $0.05 \mathrm{M}$ glycine buffer ( $\mathrm{pH}$ 9.8) $0.65 \mathrm{ml}$ was incubated at $30^{\circ}$ for $60 \mathrm{~min}$, and optical densities at $340 \mathrm{~m} \mu$ before and after the reaction were measured. An assay curve obtained by using concentrations less than $5 \times 10^{-3} \mathrm{M}$ mannitol (authentic) was used as a standard.

Measurement of $\mathrm{CO}_{2}$-fixation capacity. (1) $\mathrm{CO}_{2}$-fixation in water. Vessels used were a shaking flask $(500 \mathrm{ml})$ and an Erlenmyer flask $(100 \mathrm{ml})$ with rubber caps, which contained $90 \mathrm{ml}$ and $20 \mathrm{ml}$ of conidia suspensions, respectively. To the conidia suspension $(0.5 \mathrm{mg}$ dry weight of conidia $/ \mathrm{ml})$ was added $\mathrm{Na}_{2}{ }^{14} \mathrm{CO}_{3}$ solution to give a final radioactivity of $1 \mu \mathrm{c} / \mathrm{ml}$ and the suspension was incubated at $30^{\circ}$ with shaking.

Samples taken at intervals were collected and washed on membrane filters (Membranfiltergeselschaft Nr. 1) and radioactivities of the conidia samples were directly measured with a thin-window Geiger-Müller counter.

The conidia samples of $4.5 \mathrm{ml}$ aliquots were fractionated by the modified

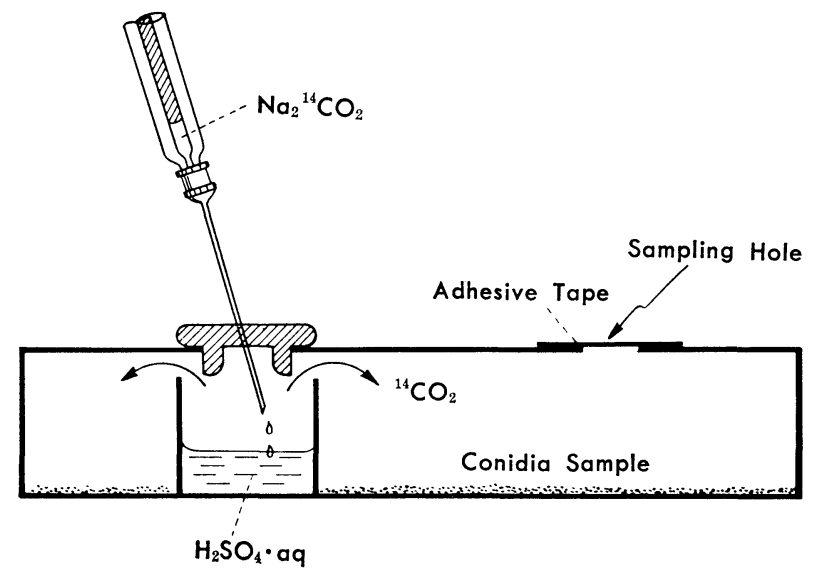

Fig. 1. Vessel designed for storing powdery conidia in the presence of ${ }^{14} \mathrm{CO}_{2}$ at a constant relative humidity.

For procedure see text. 
method of SchmidT and Thannhauser $(6)$, and the radioactivity of each fraction was counted with a windowless gas-flow counter.

(2) $\mathrm{CO}_{2}$-fixation in moist air. When the activity of $\mathrm{CO}_{2}$-fixation of powdery conidia in the moist air was measured, a vessel designed for this purpose (Fig. 1) was employed. A closed, flat and cylindrical vessel $(63 \mathrm{ml}$ in volume) made of plastic was assembled with an inner well containing sulfuric acid solution which kept the relative humidity in the vessel at $94 \%$. To this solution was added a small amount of $\mathrm{Na}_{2}{ }^{14} \mathrm{CO}_{3}$ through a rubber cap with a microsyringe to give a final concentration of ${ }^{14} \mathrm{CO}_{2}$ at $10 \mu \mathrm{c} / \mathrm{ml}$ in the atmosphere. A sampling hole on the top was covered with two sheets of an adhesive polyethylene tape and an aliquot of the conidia sample spread over the bottom was quickly taken out at intervals with a microspatula at the sampling hole. The sampling hole cover was lifted only enough to permit sampling. The conidia sample was suspended in water and collected on a membrane filter to measure radioactivity as mentioned above.

Chemical analyses. The protein content in conidia was measured by the method of LOWRY et al. (7) and RNA content by the orcinol method (8).

\section{RESULTS}

\section{Storage in gases}

Conidia samples stored for various periods at $30^{\circ}$ in an atmosphere of $94 \%$ relative humidity were cultured on the germination media and the courses of germination were followed. As seen in Fig. 2, conidia stored for 51 days germinated almost completely a little later than the fresh control, whereas those stored for more than 80 days germinated only partially and with an appreciable delay. The following data show the germination rates determined after $5.5 \mathrm{hr}$ of cultivation on the germination medium.

The effect of variation of humidity in the presence and absence of oxygen on the longevity of conidia was examined. As shown in Fig. 3a, when the course of conidia deterioration in air and nitrogen of higher humidities were compared, deterioration proceeded more rapidly under anaerobiosis than under aerobiosis. However, if the atmosphere was kept almost completely dry, conidia were stable for more than 96 days at $30^{\circ}$ even under anaerobiosis. In general, the course of deterioration in each case was not linear, but there appeared a lag prior to a decrease in germinating ability. Fig. $3 \mathrm{~b}$, redrawn from Fig. 3a, indicates the relationship between the relative humidity and the germination rate of conidia stored for 96 days. It is clear that the conidia were rather unstable in air at relative humidity of more than $70 \%$, while in nitrogen the response to the humidity became much more sensitive.

When the gas phase of the vessel was filled with air containing various concentrations of $\mathrm{CO}_{2}(0-0.3 \%)$, no conspicuous effect of $\mathrm{CO}_{2}$ was observed 


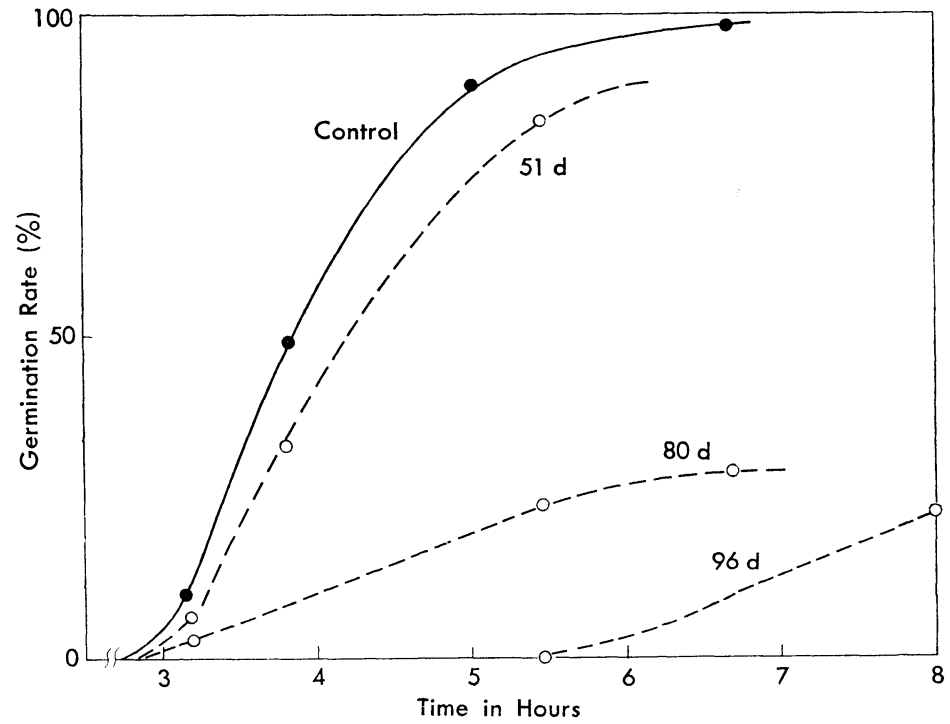

Fig. 2. Germination processes of conidia stored in moist air.

Powdery conidia were stored at $30^{\circ}$ in air of $94 \%$ relative humidity, and the samples taken at intervals were tested for their germinative capacities. Figures indicate the duration of storage in days. In control experiments, fresh conidia stored dry in the cold were employed.
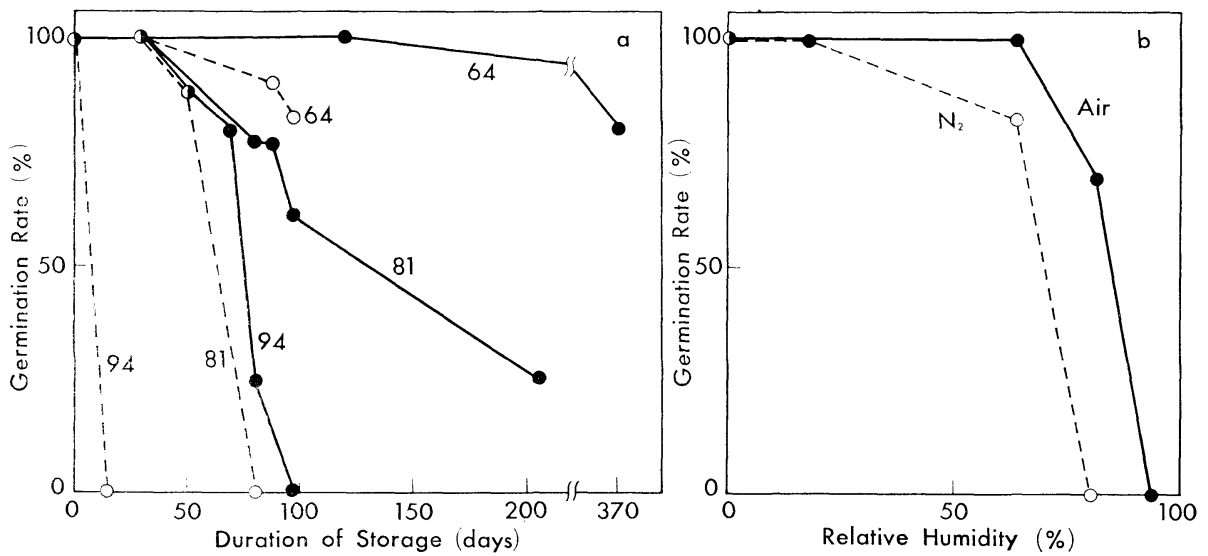

Fig. 3. Changes in germinative capacity of conidia as stored in air and in nitrogen at different relative humidities.

- in air, - - - - - in nitrogen.

a: Time courses of changes in germinative capacities of powdery conidia during storage. Figures indicate relative humidities in per cent.

b: Relationship between relative humidity and germination rate of conidia stored for 96 days (redrawn from a). 


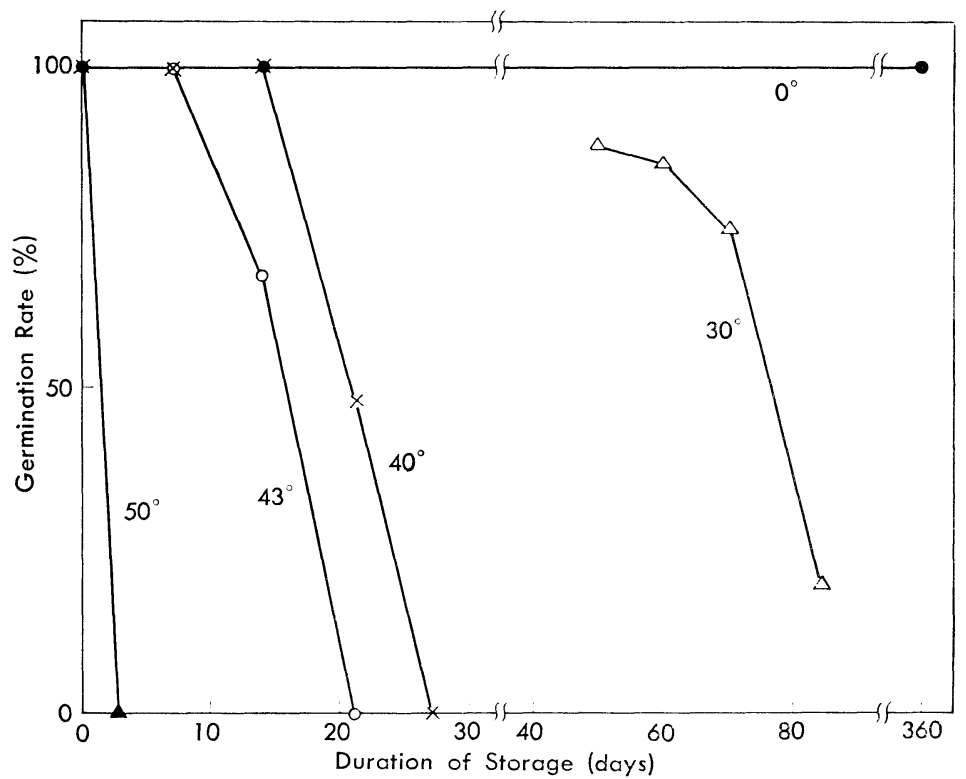

Fig. 4. Time courses of changes in germinative capacities of conidia stored at different temperatures.

Powdery conidia were stored in air of $94 \%$ relative humidity at different temperatures indicated in the figure.

on the longevity of conidia.

When conidia were stored in air of $94 \%$ relative humidity at various temperatures, the germination rate changed as shown in Fig. 4. In this case also the deterioration of conidia occurred after a lag period. For the determination of deterioration rate, two parameters were taken: (1) the rate for entering into the course of decrease in germination rate (the reciprocal of duration of lag period), and (2) the rate of decrease in germination rate. Fig. 5 shows the relation between the reciprocal of absolute temperature $(1 / T)$ and the logarithm of the rate of conidia deterioration. It is clearly indicated in this figure that the two rates change almost in parallel with each other against $1 / T$ and that there are breaks in their relations at around $40^{\circ}$. These observations suggest that (1) the length of the lag period is inversely related to the rate of decrease in germination rate, and (2) the processes of conidia deterioration above and below $40^{\circ}$ are apparently different in nature.

\section{Storage in water}

Fig. 6 a shows the time courses of germination of conidia stored in water under shaking for different periods. The prolongation of germination 


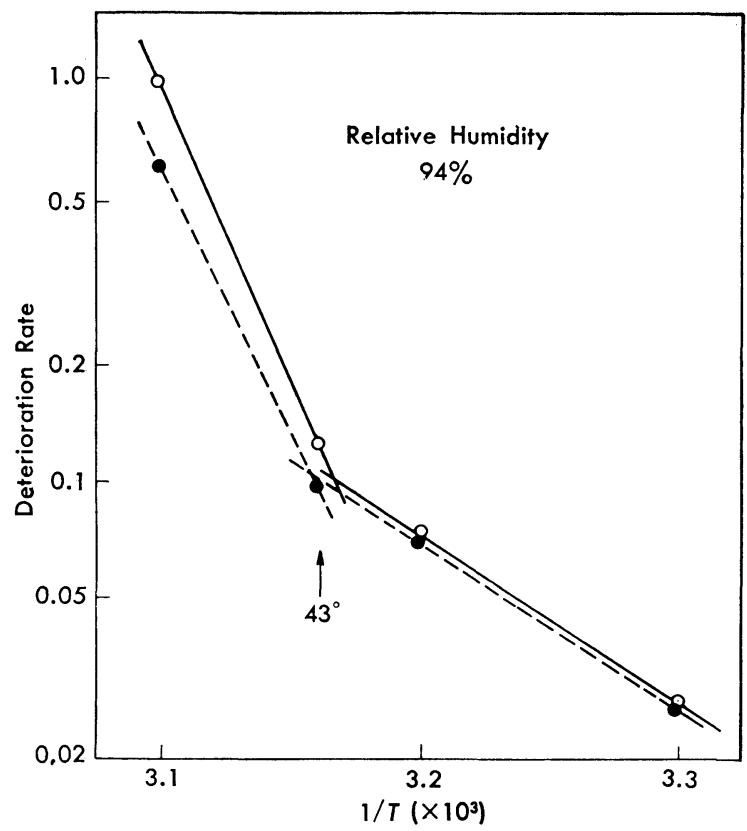

Fig. 5. Arrhenius plot showing the relationship between temperature and rate of conidia deterioration.

- - Deterioration rate 1 , representing the rate for entering into the course of loss of germinative capacity ( 1 /duration of lag period).

--- - - Deterioration rate 2, representing the rate of decrease in germination rate $(\Delta$ germination rate/day/100).

time and the loss of germinative ability during storage were, in principle, similar to those shown in Fig. 2. Characteristic in this case was the acceleration of germination observed in conidia stored for less than 10 days.

The result of similar experiment is also shown in Fig. 6b, in which the ordinate indicates the percentage of decrease (minus region) and increase (plus region) of $50 \%$ germination time as compared with that of control conidia. The decrease of germination time occurred when conidia were stored in water for less than ca 10 days, whereas the increase was observed in conidia stored for more than 40 days. It is interesting to note that those conidia stored for 15-30 days germinated almost simultaneously with control conidia. The germination was somewhat accelerated when conidia were incubated in water for only $1 \mathrm{hr}$ at $30^{\circ}$, but not at $0^{\circ}$. This indicates that some part of the germination reaction has proceeded even in water during such a short period. 

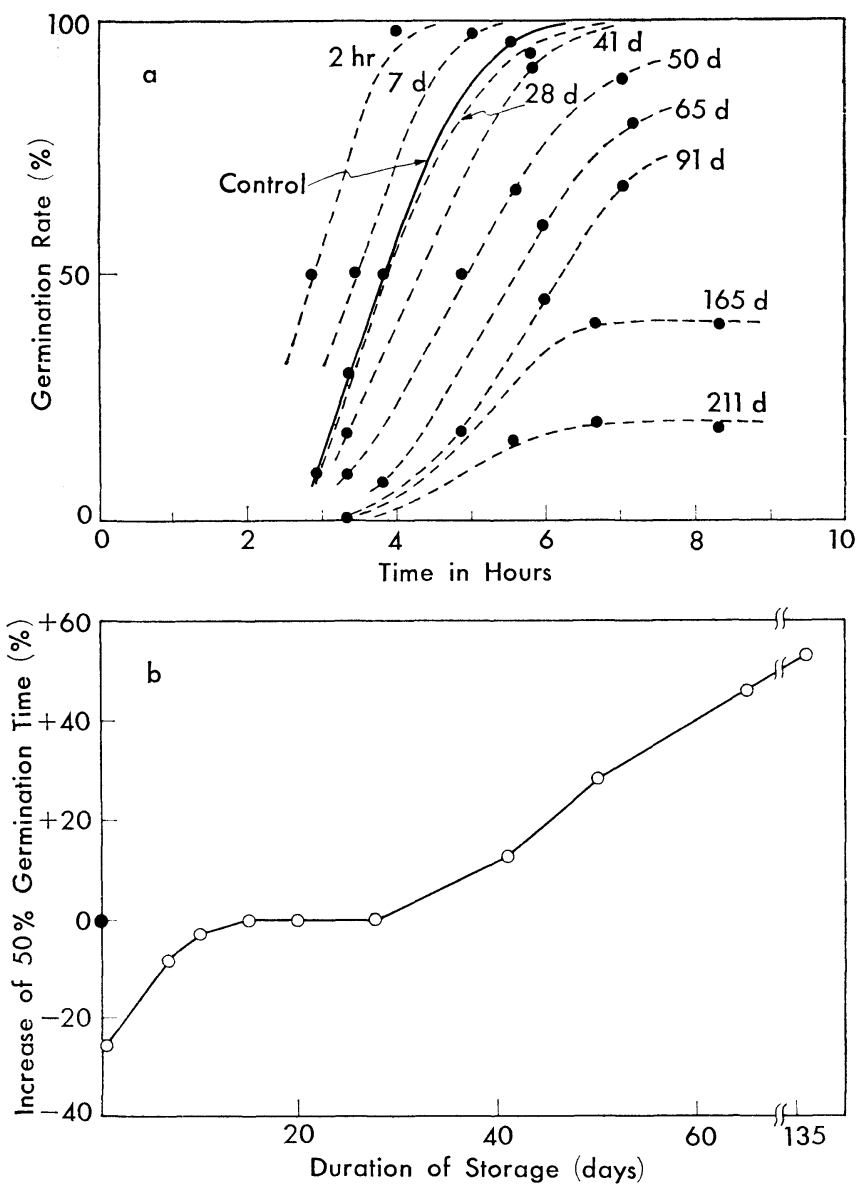

Fig. 6. Changes in germinative capacities of conidia stored in water at $30^{\circ}$.

a: Germination processes of conidia sample stored in water for different periods. Figures indicate durations of storage. For control see legend of Fig. 1.

$\mathrm{b}$ : Changes in $50 \%$ germination time of conidia samples during storage in water. Length of $50 \%$ germination time of control conidia was taken as zero and percentage increase (plus sign) and decrease (minus sign) of 50\% germination time were plotted for each conidia sample.

When the conidia which had been stored in water for 7 days were dried in vacuo and stored for 450 days in dryness at $4^{\circ}$ and $30^{\circ}$, they germinated to the extent of $77 \%$ and $27 \%$, respectively. Since unwashed powdery 
conidia stored in dryness for more than 1 year at $30^{\circ}$ could germinate almost completely as mentioned earlier, it may be concluded from the above result that those conidia once induced for germination in water deteriorate to some extent at $30^{\circ}$ even in the absence of water.

\section{Effect of physiological state of conidia on longevity}

To compare the longevity of dormant and partially germinated conidia, those samples taken at varied germination steps were washed and stored by suspending them in water. When these conidia samples taken at intervals during the course of the storage were tested for germinative capacity, partially germinated conidia were found to deteriorate much faster than the control dormant conidia (Fig. 7). It seems likely that the longevity of conidia is largely dependent on the physiological state (i.e., dormant and partially germinated states) of conidia to be stored.

Direct comparison of various storage conditions affecting longevity

In the aforementioned observations, each set of experiments was carried

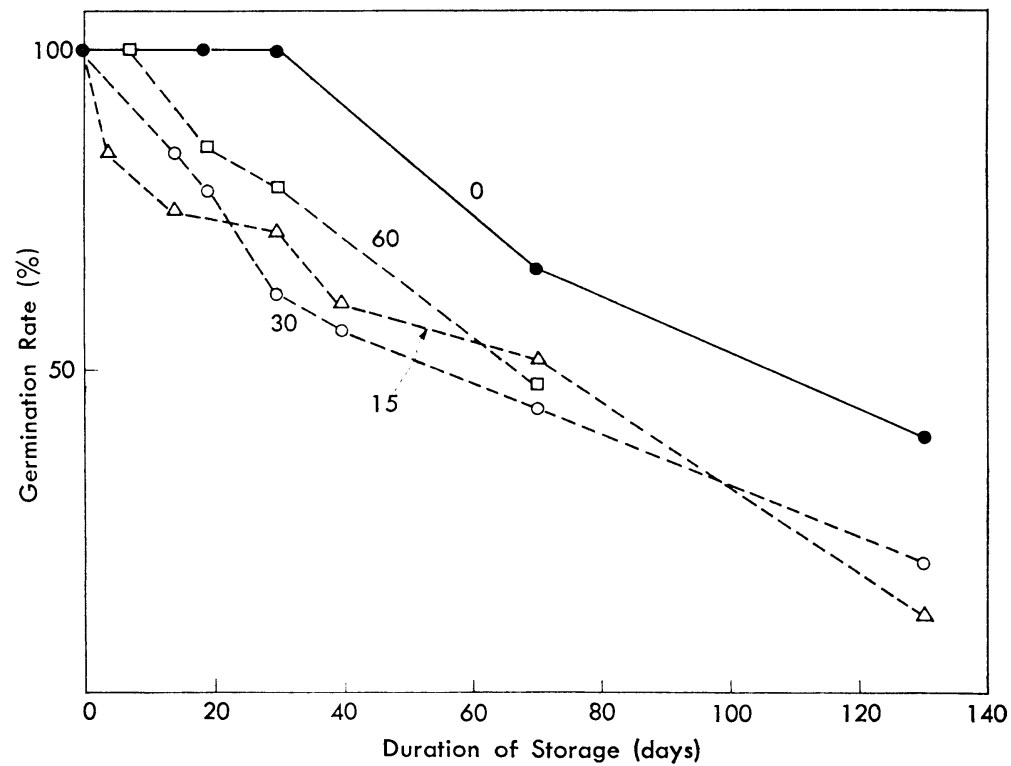

Fig. 7. Time courses of decrease in germinative capacities of partially germinated conidia samples during storage in water.

Conidia samples had previously been cultured for different periods in germination media at $30^{\circ}$, washed and stored in water by shaking at $30^{\circ}$. Samples taken at intervals after the transplantation in water were tested for their germinative capacities.

Figures indicate durations of precultivation in minutes. 'Zero' sample represents untreated control conidia. 
out separately. In an attempt to obtain clear-cut comparable results of the effects of various storage conditions on the conidia longevity, conidia samples of the same batch were stored under different conditions and changes in germinative capacity during storage were followed. Conditions of storage selected were: (1) in dry air at $4^{\circ}$ as a powdery form, (2) in moist air (94\% relative humidity) at $30^{\circ}$ as a powdery form, (3) in water at $30^{\circ}$ after repeated washings of dormant conidia, and (4) in water at $30^{\circ}$ after partial germination ( $15 \mathrm{~min}$ cultivation in the germination medium) of conidia.

As clearly indicated in Fig. 8, those conidia stored dry at $0^{\circ}$ were completely stable after 210 days of incubation. It is worth mentioning that dormant conidia stored in water and moist air entered into the course of deterioration almost simultaneously after 30 days, but the rate of deterioration was much slower in the former than in the latter. The partially germinated conidia stored in water entered into the course of deterioration much faster than dormant conidia stored in water, although the courses of

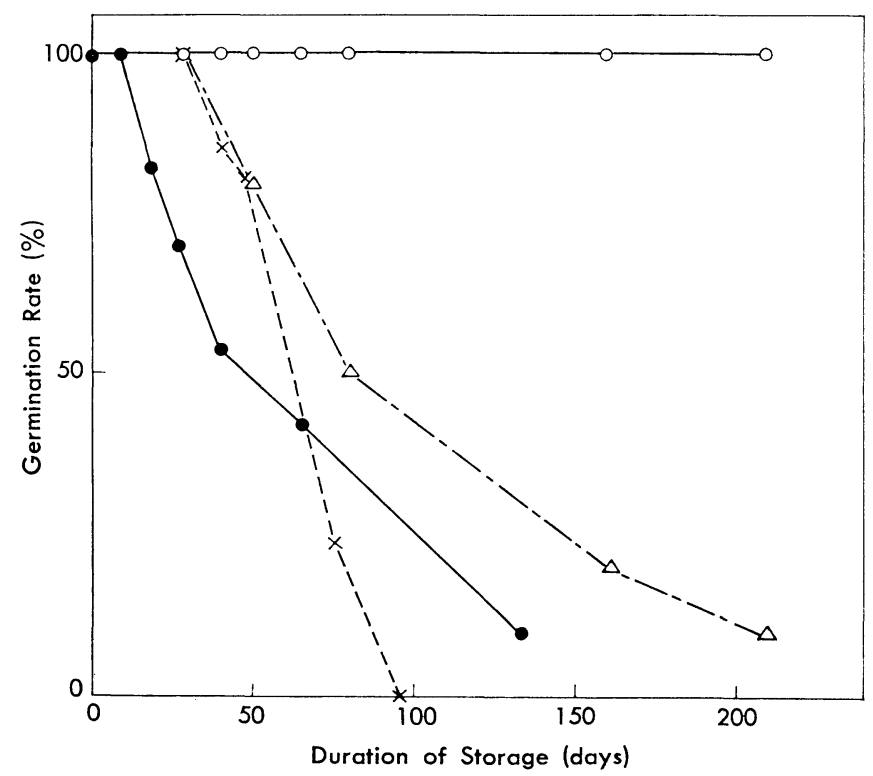

Fig. 8. Direct comparison of time courses of conidia deterioration under different storage conditions.

- $\mathrm{O}-$ Powdery conidia stored in dry air at $4^{\circ}$.

- - $\times---$ Powdery conidia stored in moist air $194 \%$ relative humidity) at $30^{\circ}$.

$-\triangle-$ Washed conidia stored in water at $30^{\circ}$.

- Partially germinated $(15 \mathrm{~min}$ precultivated) conidia stored in water at $30^{\circ}$. 
the deterioration of the two samples were almost in parallel with each other after the germinative capacity began to decrease.

Change in respiratory activity of conidia during storage

To examine the physiological changes of conidia during storage, the respiratory activity of conidia stored in water was measured. Since the respiratory activity of conidia was very low, we employed an oxygen electrode for the measurement of oxygen consumption.

Fig. 9a indicates courses of consumptions of dissolved oxygen by various conidia samples of different agings. In these experiments, glucose was added to the measurement system after $20 \mathrm{~min}$. In Fig. $9 \mathrm{~b}$ are shown the $\mathrm{QO}_{2}$ changes of endogenous and glucose respirations of conidia during storage. The endogenous respiration of dormant conidia decreased gradually for nearly 40 days, and finally no endogenous activity was detected by the present method after 50 days of storage. It should be noticed that when the endogenous respiratory activity was lost, conidia entered the course of deterioration (see Fig. 7). By contrast, the glucose respiration increased gradually for about a week and the high value was maintained even after 90 days of storage, when the germination rate was nearly $50 \%$.

Our previous work (9) suggested that mannitol is a possible substrate for the endogenous respiration of dormant conidia, since it was present in conidia in large amounts (10.6\% of dry weight of conidia). Therefore, the course of change in amount of mannitol during the storage in water was followed. The mannitol content in conidia decreased gradually as the time of storage proceeded. However, when the endogenous respiratory activity became undetectable at around 50 days of storage, mannitol was still present in an amount of ca $70 \%$ of the initial content. Thus mannitol does not appear to be necessarily the sole substrate of endogenous respiration. Instead, the mannitol content seemed to be almost proportional to the germinative capacity of the stored conidia (Fig. 10). It should be added that at the 50th day of storage, ribonucleic acid and protein contents in conidia were $95 \%$ and $100 \%$ of the initial contents, respectively.

\section{Relationship between $\mathrm{CO}_{2}$-fixation capacity and aging of conidia}

It has been ascertained in this laboratory that the $\mathrm{CO}_{2}$ is one of the essential substrates for germination (10) and that $\mathrm{CO}_{2}$-fixation in conidia is one of the most characteristic events during the initial step of germination $(11,12)$. In an attempt to relate such findings to the aging of conidia, the activity of ${ }^{14} \mathrm{CO}_{2}$-uptake in buffer by aged conidia samples stored under moist air and in water were compared.

The courses of ${ }^{14} \mathrm{CO}_{2}$-uptake by the control conidia and aged ones stored for 203 days under the moist air (81\% relative humidity), which germinated to the extent of only $20 \%$ after $5.5 \mathrm{hr}$, were followed. Fig. 11 indicates that the aged conidia took-up ${ }^{14} \mathrm{CO}_{2}$ even more actively than the fresh conidia. 

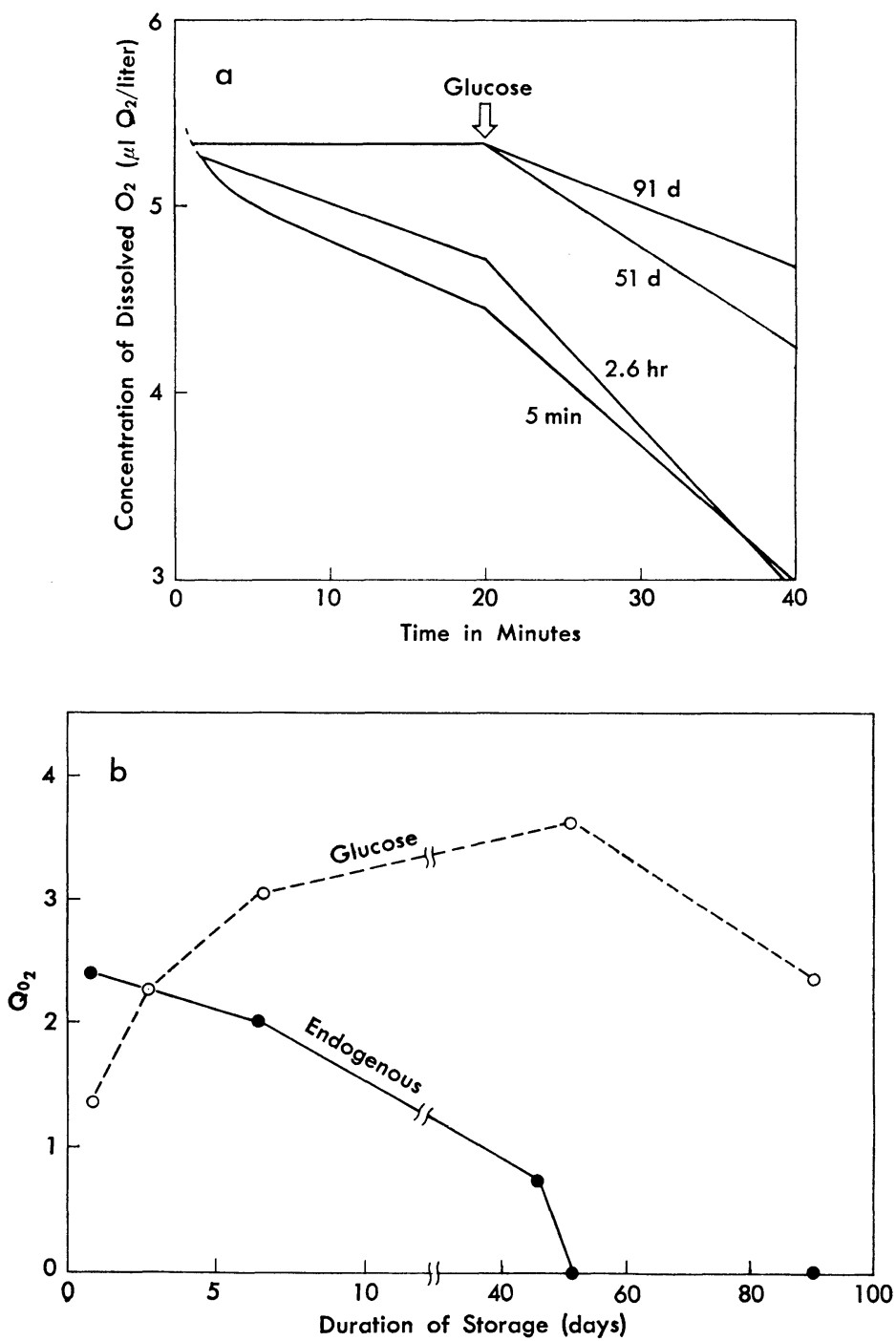

Fig. 9. Changes in respiratory activities of conidia samples during storage in water.

a: Several examples of time courses of consumption of dissolved oxygen in water by conidia samples stored in water at $30^{\circ}$ for different periods. After $20 \mathrm{~min}$, glucose was added. Details of procedure are described in MATERIALS AND Methods.

$\mathrm{b}$ : Changes in endogenous and glucose respiration activities of conidia during storage in water at $30^{\circ}$. 


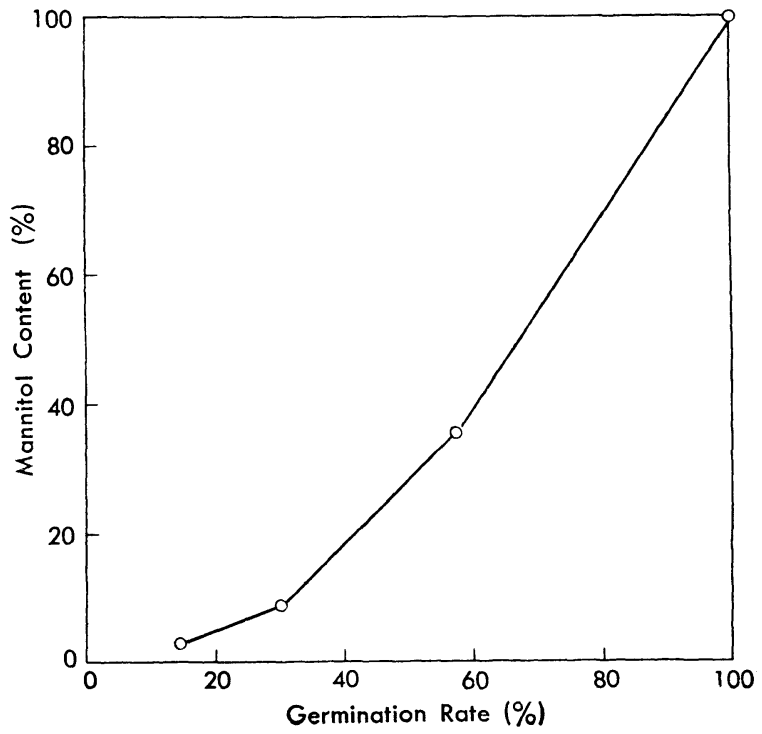

Fig. 10. Relationship between germinative capacity and mannitol content of conidia shaken in water.

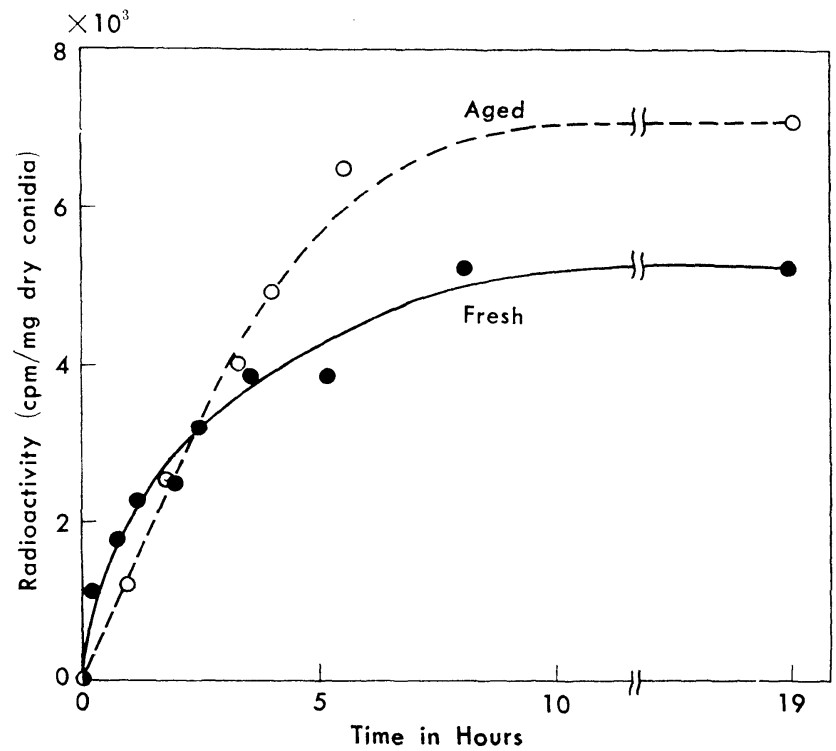

Fig. 11. Comparison of ${ }^{14} \mathrm{CO}_{2}$-fixation activities of fresh and aged conidia.

Powdery conidia stored in moist air ( $81 \%$ relative humidity) for 203 days and fresh ones were suspended in water containing ${ }^{14} \mathrm{CO}_{2}$ and the course of ${ }^{14} \mathrm{CO}_{2}$-uptake into intact conidia were followed. 
Further experiment revealed as shown in Fig. 12 that in the aged conidia the incorporation of ${ }^{14} \mathrm{CO}_{2}$ is primarily into low molecular weight substances (the $50 \%$ ethanol soluble fraction) but not into high molecular weight substances, such as protein and nucleic acids. This observation implies that aged conidia stored in water for a long time are able to fix $\mathrm{CO}_{2}$ but not to assemble biopolymers.

Another experiment employing the powdery conidia stored in moist air was performed to examine whether or not the conidia are capable of $\mathrm{CO}_{2}$ fixation during storage. A vessel made of a plastic (Fig. 1) was used for this purpose. Appreciably no incorporation of ${ }^{14} \mathrm{CO}_{2}$ into conidia occurred during 30 days of storage. This suggests that in powdery conidia stored in moist air the germination process was not induced to proceed.

In contrast to conidia stored in moist air, those stored in water took-up ${ }^{14} \mathrm{CO}_{2}$ only during the initial $8 \mathrm{hr}$ of storage as clearly indicated in Fig. 11 (see the curve denoted as "fresh"). Such conidia stored in water for more than $10 \mathrm{hr}$ were incapable of $\mathrm{CO}_{2}$-fixation.

\section{DISCUSSION}

The longevity of fungal spores varies from species to species. Among spores of various fungi, conidia of certain imperfect fungi are known to survive for very long periods. A. oryzae conidia used in the present investigation also survived in a completely active form for years under dry condition in a refrigerator.

Relative humidity is one of the major factors in spore longevity. According to Cochrane (2), modes of response of fungal spores to atmospheric moisture may be divided into three different types: (1) spores survive longest at saturation, (2) spore survival is greatest at intermediate humidity and is less at either extreme, and (3) spores remain viable longest at low humidity. A. oryzae conidia belong to the last type. Although the conidia remained survived for nearly one year at $0^{\circ}$ even under high humidity, they lost germinating activity at $30^{\circ}$ rather rapidly in humid air and especially rapidly in humid nitrogen gas. This seems to support the earlier view that at high humidity the metabolism of such fungal spores is accelerated with a consequent reduction in longevity (13).

Temperature effect on the conidia longevity is worthy of notice. Deterioration rate and temperature relation showed that the nature of deterioration at moderate and higher temperatures differs thermodynamically at around $40^{\circ}$. A rapid deterioration occurring at higher temperatures may be caused by the thermal denaturation of certain enzymes, while a slow deterioration at moderate temperatures may result from some unknown physiological processes.

Deterioration of conidia in water differs from that in moist air in that the acceleration (decrease) of germination time occurred initially during 


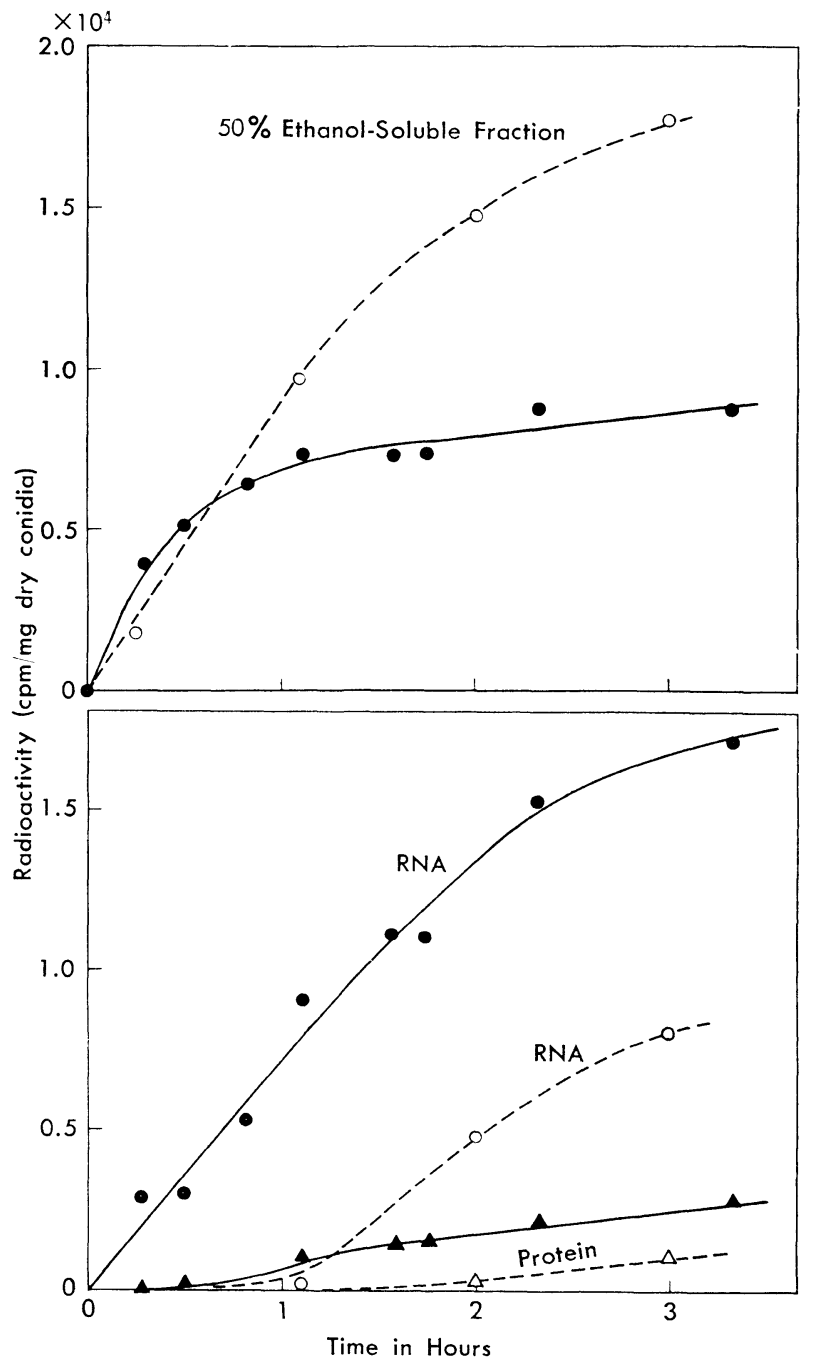

Fig. 12. Comparison of ${ }^{14} \mathrm{CO}_{2}$-incorporation into various fractions of fresh and aged conidia.

Fresh and aged conidia (see legend of Fig. 11) were suspended in water containing ${ }^{14} \mathrm{CO}_{2}$ and conidia samples taken at intervals were fractionated essentially by the method of SchmidT-ThANNHAUSER and each fraction subjected to radioactivity measurement.

Solid and broken lines represent fresh and aged conidia, respectively. 
storage. This seems to be an important point in consideration of the mechanism of deterioration. As reported recently by ONo et al. (12), $A$. oryzae conidia could synthesize a certain molecular species of RNA even in water or in phosphate buffer in which neither swelling nor germination of conidia occurred. The RNA synthesis continued for several hours and finally leveled off as compared with normally germinating conidia, in which harmonized syntheses of various RNA molecules followed after the initial synthesis of a certain molecular species of RNA's similar to those synthesized in water. Therefore, the acceleration of germination time occurring during the early period of storage in water seems to be caused by a partial induction of metabolic process(es) relating to germination. This indicates that the deterioration of conidia in water ensues after a certain initial process (step A) of germination as schematically indicated in Fig. 13 with thin arrows. If the germination reaction proceeds further (see step B in Fig. 13) as demonstrated by the precultivation of conidia in the germination medium, the longevity of such conidia in water is shortened significantly. Thus the advancement of growth metabolism affects unfavorably on the maintenance of conidia viability.

Does the deterioration of powdery conidia in moist air proceed through the same process? Comparative experiments on the $\mathrm{CO}_{2}$-fixation activities of conidia stored in water and in moist air may partly answer the question. As reported earlier (14), the conidia cannot germinate in the absence of $\mathrm{CO}_{2}$ even in a complete germination medium. This indicates that $\mathrm{CO}_{2}$ is one of the essential substrates for the induction of germination. It was indeed shown that germinating conidia took up ${ }^{14} \mathrm{CO}_{2}$ actively without lag in the very initial phase of germination and radioactivity was found in the

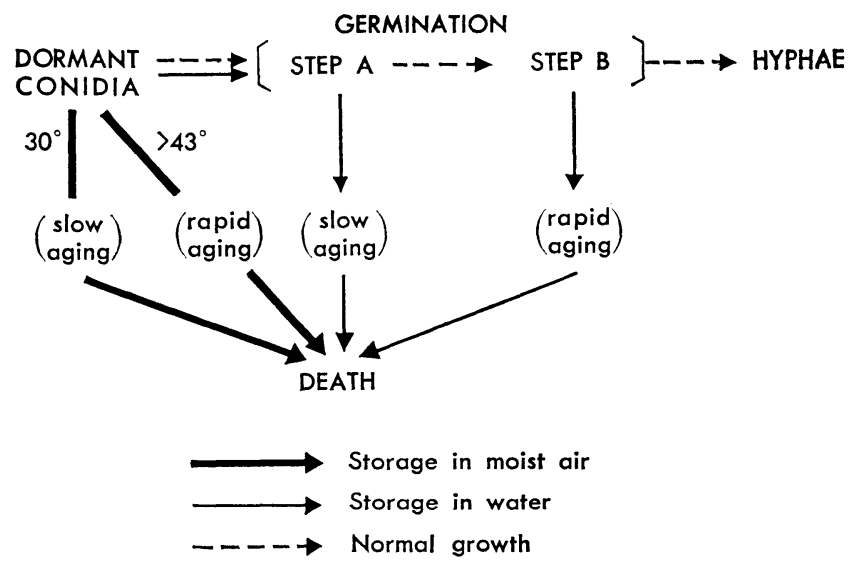

Fig. 13. Schematic representation of mode of deterioration of Aspergillus oryzae conidia under various conditions. 
RNA fraction after only 3 min of cultivation (10-12). In the present experiments, when conidia were stored in water, ${ }^{14} \mathrm{CO}_{2}$ was found to be incorporated into conidia for the first several hours, and after this period, ${ }^{14} \mathrm{CO}_{2}$ was no longer incorporated. By contrast, when the conidia were stored in moist air containing ${ }^{14} \mathrm{CO}_{2}$, no incorporation of the tracer was observed for a long period. These observations clearly indicate that in conidia stored in water a partial induction of a certain germination reaction detectable with the use of ${ }^{14} \mathrm{CO}_{2}$ occurs, whereas in conidia stored in moist air no such reaction proceeds as schematically indicated in Fig. 13 with bold arrows. The fact that appreciably no acceleration of germination was observed during the storage of conidia in moist air may strongly support the present view. It should be mentioned that conidia stored in moist air for a long period were able to take up ${ }^{14} \mathrm{CO}_{2}$ when suspended in water and the incorporation of ${ }^{14} \mathrm{CO}_{2}$ occurred mainly into low molecular weight substances and not into biopolymers as compared with fresh conidia in water. This suggests that in the former conidia sample some enzymes involved in biopolymer formation may be impared during the storage.

As seen in many observations on the course of conidial aging, the course of deterioration (decrease in germinative capacity) started after a lag or stable period, and the duration of the lag was found to be inversely proportional to the rate of deterioration. The present observation suggests that the duration of the lag period may be closely related to the respiratory activity of the conidia under storage.

As already known, dormant conidia of Aspergillus have weak respiratory activity. When changes in activities of endogenous and substrate respirations were examined, the activity of endogenous respiration remained almost constant until some time before the onset of decrease in germinative capacity, while the respiratory activity with glucose as substrate remained almost unchanged even after the deterioration proceeded considerably. Worth noticing is the fact that the course of deterioration started after a rather rapid loss of endogenous respiratory activity. This suggests that during the lag period, intracellular events leading to the cell deterioration are suppressed by some energy consuming mechanism, and after the endogenous substrate for respiration has been consumed, conidia may get into the course of deterioration.

From such inference, a question arises: what is the substrate for the endogenous respiration? One of the more probable substrates is mannitol contained in the conidia in large amounts. The present experiments indicated that only $30 \%$ of total mannitol had been consumed when the activity of endogenous respiration became undetectable. From this result, one is tempted to conclude that mannitol may not be the substrate limiting the activity of endogenous respiration. However, the recent finding of HORIKOSHI et al. (5) showed that about $22 \%$ of total mannitol in A. oryzae conidia was consumed in the initial $1 \mathrm{hr}$ of germination and then its content in- 
creased attaining its original level. This implies that a $20-30 \%$ fraction of intracellular mannitol may play a special role in the early phase of germination and that, when the conidia are stored in water, the same fraction of mannitol may be utilized as substrate for the maintenance of the "stable period" during storage. At any rate, the conidia longevity seems to be highly dependent on mannitol content as demonstrated by the proportionality between these two parameters.

\section{SUMMARY}

Fresh and dry conidia of Aspergillus oryzae were examined for their longevity under various storage conditions by testing germination rate at intervals over a year.

Powdery conidia were stable for years when they were kept dry at $4^{\circ}$. Conidia stored in moist air (more than 70\% relative humidity) survived for a few months, while those stored in moist nitrogen gas for less than a week. Washed conidia stored in water by shaking survived a little longer than powdery conidia stored in moist air.

Characteristic difference between the storage of conidia in water and in moist air was that in the former case, but not in the latter, an acceleration of germination was observed at the initial phase of storage. The acceleration seemed to be caused by the fact that germination reaction proceeds partially even in water as demonstrated by ${ }^{14} \mathrm{CO}_{2}$-incorporation into biopolymers.

Deterioration of conidia appeared to be partially dependent on the consumption of mannitol, an intracellular storage substance. Modes of deterioration processes under different storage conditions were discussed based on the observations on respiratory and biosynthetic activities of conidia.

Authors' thanks are due to Dr. T. Nakamura of University of Tokyo, who gave them valuable advise in the measurement of respiratory activity with an oxygen electrode. Senior author (M.T.) expresses her heartfelt gratitude to Dr. T. Otsuki and Dr. J. Dan of Ochanomizu University for their encouragements throughout the course of the present investigation.

\section{REFERENCES}

1) L. E. HAwker: Physiology of Fungi, University of London Press, London (1950), p. 217.

2) V. W. Cochrane: Physiology of Fungi, John Wiley \& Sons, London (1958), p. 398.

3) G. Terui and T. Mochizuki: Technol. Rept. Osaka Univ., 5, 219 (1955).

4) C. D. Hodgman, R. C. Weast and C. W. Wallace: Hand Book of Chemistry and Physics, 35th ed., Chemical Rubber Pub. Co., Cleveland (1953), p. 2310.

5) K. Horikoshi, S. IIdA and Y. IkedA: J. Bacteriol., 89, 326 (1965). 
6) A. Nishi: J. Bacteriol., 81, 10 (1961).

7) O. H. Lowry, N. J. Rosebrough, A. L. Farr and R. J. Randall: J. Biol. Chem., 193, 265 (1951).

8) E. Volkin and W. E. Cohn: Methods of Biochemical Analysis, ed. by D. Glick, Vol. I., Interscience Pub., New York (1954), p. 287.

9) I. Takebe: J. Gen. Appl. Microbiol., 6, 83 (1960).

10) T. Yanagita: J. Gen. Appl. Microbiol., 9, 343 (1963).

11) Y. Tsay, A. Nishi and T. Yanagita: J. Biochem., Tokyo, 58, 487 (1965).

12) T. Ono, K. Kimura and T. Yanagita: J. Gen. Appl. Microbiol., 12, 13 (1966).

13) E. L. Merek and C.L. Fergus: Phytopathol., 44, 54 (1961).

14) I. Takebe and N. Shimizu: Ann. Rept. Inst. Food Microbiol., Chiba Univ., 12, 29 (1959). 